\title{
Simulation of the fate and transport of boron nanoparticles in two-dimensional saturated porous media
}

\author{
Chunmei Bai ${ }^{1,2, *}{ }^{\infty}$, Baisha Weng ${ }^{3}$ and Huan Sheng Lai ${ }^{4}$ \\ ${ }^{1}$ Shenzhen Institutes of Advanced Technology, Chinese Academy of Sciences, Shenzhen 518055, \\ People's Republic of China. \\ ${ }^{2}$ Shenzhen Audaque Data Technology Co. Ltd., Shenzhen 51805\%, People's Republic of China. \\ ${ }^{3}$ State Key Laboratory of Simulation and Regulation of Water Cycle in River Basin, China Institute of Water \\ Resources and Hydropower Research, Beijing, People's Republic of China. \\ ${ }^{4}$ College of Chemical Engineering, Fuzhou University, Fuzhou 350108, Fujian, People's Republic of China. \\ ${ }^{*}$ Corresponding author. e-mail: baichunmei2005@163.com
}

MS received 24 June 2017; revised 14 November 2017; accepted 14 January 2018; published online 1 December 2018

The wide production and application of engineered nanomaterials (ENMS) inevitably lead to their release in the groundwater environment. However, the release and transport of boron nanoparticles in the multi-dimensional subsurface remain largely unknown. In this work, a multi-dimensional numerical simulator for the transport of boron nanoparticles in the saturated porous media was first developed and validated. Hypothetical scenarios for the release of boron nanoparticles into a layered two-dimensional (2D) and heterogeneous 2D saturated porous media were then explored, and compared with the fullerene nanoparticles. The results demonstrated that the soil heterogeneity influenced the fate of nanoparticles, with high permeable layers and high aqueous-phase concentration. Besides, the boron nanoparticles tend to accumulate at the inlet zones, where it was closer to a nanoparticles source. Different layers of interface interaction also impact the concentration of nanoparticles. In general, the mobility and aqueous-phase concentration of fullerene nanoparticles were higher than those of the boron nanoparticles. In addition, the mobility of boron nanoparticles was found to be sensitive to release concentration, soil porosity and nanoparticle aggregate size.

Keywords. Boron nanoparticles; fullerene nanoparticles; mobility; subsurface; heterogeneity.

\section{Introduction}

According to an estimate by the United Nations Environment Programme (UNEP 2007), the production of engineered nanomaterials (ENMs) may reach 58,000 tons/yr by 2020. Due to the large production of ENMs in the current market (LuxResearch 2009; WWICS 2012; Joo and Zhao 2016) and their corresponding wide applications in various areas, such as optics, medicine, cosmetics, electronics, manufacturing and biomedical technology (Mauter and Elimelech 2008), their release into the environment, especially the subsurface, has already attracted great environmental concern. A number of studies have investigated the aquatic toxicity and antimicrobial properties of ENMs and their potential effects on human health (Nakajima et al. 1996; Kamat et al. 2000; Saiers and Ryan 2005; Lyon et al. 2006). For instance, fullerene nanoparticle suspensions were found to be toxic 
to juvenile largemouth bass and human cell lines (Oberdorster 2004; Sayes et al. 2005). Besides, due to their high sorption capacity of nanomaterials, ENMs could serve as carriers for toxic substances such as polycyclic aromatic hydrocarbons (Cheng et al. 2005; Yang et al. 2006). Potentially, ENMs may enter the water supply through a number of pathways, for instance, groundwater recharge with wastewater containing nanomaterials, agricultural use of biosolids containing ENMs, etc. After entering the environment, ENMs can interact with natural organic matter, resulting in stable aggregates that can travel very long distances, even as far as drinking water (Chen and Elimelech 2007; Espinasse et al. 2007; Hotze et al. 2010). To determine the risks associated with these emerging contaminants, it is important to understand the mobility of ENMs in the subsurface saturated porous media.

Due to the variations in the properties of nanomaterials and subsurface environment, the fate and transport of ENMs in the subsurface is a case-by-case issue. Boron nanoparticles, as a promising solid fuel and propellant (Liu et al. 2009), are widely used in military and civilian areas. The wide applications of boron nanoparticles would eventually lead to their release in the subsurface environment. Yet, the release and transport of boron nanoparticles in a multi-dimensional subsurface remains largely unknown. Previously, Liu et al. (2009) investigated the transport and deposition behaviours under various electrolyte concentrations and flow velocities in one-dimensional (1D) homogeneous column studies, and the experimental results show that the fate and transport of boron nanoparticles follows the classic clean-bed filtration theory (CFT model) (Liu et al. 2009). However, little information is provided regarding the transport of boron nanoparticles in the multidimensional subsurface environment, especially the heterogeneous subsurface. Furthermore, there is currently no modelling tool to facilitate the transport of boron nanoparticles in the subsurface field scale. Thus, one of the objectives of this study is to fill this research gap by exploring an appropriate numerical simulator to study the transport and retention of boron nanoparticles in the multidimensional saturated porous media.

Besides the boron nanoparticles, the fullerene nanoparticles $\mathrm{nC}_{60}$ have been chosen as the nanomaterial of comparison to help understand the differences in the transport behaviours of various nanomaterials. Fullerene nanoparticles are comparably well studied by a number of researchers in 1D homogeneous columns (Oberdorster 2004; Cheng et al. 2005; Sayes et al. 2005; Chen and Elimelech 2006; Yang et al. 2006; Wang et al. 2008a, b). Based on various column experimental studies, Li et al. (2008) developed a 1D mechanistic model by incorporating a maximum retention capacity term to classic filtration theory, called maximum retention model ( $\mathrm{Li}$ et al. 2008), which was able to simulate the transport of $\mathrm{nC}_{60}$ in 1D columns packed with various size fractions of Ottawa sands and flow rates ( $\mathrm{Li}$ et al. 2008; Wang et al. 2008a). Later on, the model was extended to 2D and 3D numerical models (Cullen et al. 2010; Bai and Li 2012, 2014; Bai et al. 2013) to investigate the fate and transport of $\mathrm{nC}_{60}$ nanoparticles in the field scale. These studies found that the fate and transport of $\mathrm{nC}_{60}$ in subsurface is sensitive to subsurface heterogeneity, dispersivity, release scenarios, collision efficiency factor, maximum retention capacity, etc. Even though there have been some numerical simulators (Cullen et al. 2010; Bai and Li 2012, 2014; Bai et al. 2013) developed to explain the transport behaviour of $\mathrm{nC}_{60}$ in the multi-dimensional field scale, there is currently no study working on the fate and transport of boron nanoparticles in the multi-dimensional heterogeneous subsurface.

Field-scale heterogeneity of subsurface plays an important role during the transport and distribution of ENMs in the subsurface. The study by Cullen et al. (2010) investigated the impact of field-scale heterogeneities on the mobility of $\mathrm{nC}_{60}$ and multi-walled carbon nanotubes (MWCNT), and found that the distribution of nanoparticles in the heterogeneous field is much less uniform in the heterogeneous field than in the homogeneous field site, with preferable pathways in more permeable zones. Also, the results demonstrated that nanoparticles were less mobile in heterogeneous systems compared to the homogeneous systems with the same averaged hydraulic properties. In another study by Bai et al. (2013), the release of fullerene nanoparticles into a mildly heterogeneous site (Bachman Road site in Oscoda, Michigan, USA) (Christ et al. 2005) and a highly heterogeneous site (Columbus Air Force Base site in Mississippi, USA) (Barlebo et al. 2004) was addressed and compared. The results showed that the transport and distribution of fullerene nanoparticles is much more uncertain and harder to predict for the highly heterogeneous site than the mildly heterogeneous site. These studies (Barlebo et al. 2004; 
Cullen et al. 2010; Bai et al. 2013) highlight the importance of subsurface heterogeneity on the transport and distribution of nanoparticles in groundwater. However, so far there is no study working on investigating the fate of boron nanoparticles in the multi-dimensional heterogeneous field site. This work would first address the impact of subsurface heterogeneity on the fate of boron nanoparticles in multi-dimensional saturated porous media, and then compare with that of nano-fullerenes $\left(\mathrm{nC}_{60}\right)$.

\section{Nanoparticle transport modelling}

Classic CFT model (Yao et al. 1971) was traditionally used to model nanoparticles transport in saturated porous media. Based on a number of 1D column studies, the work by Liu et al. (2009) also demonstrated that boron nanoparticles follow CFT model. According to the CFT model, the mass balance of boron nanoparticles in aqueous and attached phases could be described as follows:

$$
\begin{gathered}
\frac{\partial C}{\partial t}+\frac{\rho_{\mathrm{b}}}{n} \frac{\partial S}{\partial t}-\nabla(\overline{\mathbf{D}} \cdot \nabla C)+\mathbf{V} \cdot \nabla C=0 \\
\rho_{\mathrm{b}} \frac{\partial S}{\partial t}=n k_{\mathrm{att}} C \\
k_{\mathrm{att}}=\frac{3(1-n) v}{2 d_{\mathrm{c}}} \alpha \eta_{0} .
\end{gathered}
$$

Here, $C$ is the aqueous-phase concentration of boron nanoparticles $\left[\mathrm{M} / \mathrm{L}^{3}\right], S$ is the solid-phase concentration of boron nanoparticles $[\mathrm{M} / \mathrm{M}], t$ is time $[\mathrm{T}], n$ is the soil porosity, $\rho_{\mathrm{b}}$ is the soil bulk density $\left[\mathrm{M} / \mathrm{L}^{3}\right], \mathbf{V}$ is the pore water velocity vector $[\mathrm{L} / \mathrm{T}], \overline{\mathbf{D}}$ is the hydrodynamic dispersion tensor $\left[\mathrm{L}^{2} / \mathrm{T}\right], k_{\text {att }}$ is the boron nanoparticles attachment rate coefficient $[1 / \mathrm{T}]$ (Ma et al. 2009), $\alpha$ is the collision efficiency factor, $d_{\mathrm{c}}$ is the mean grain size [L] and $\eta_{0}$ is the single collector efficiency (Tufenkji and Elimelech 2004).

Currently, there is no modelling tool for the fate and transport of boron nanoparticles in the multi-dimensional subsurface. In our previous work (Bai and Li 2012), a modular 3D transport model for multi-species (MT3DMS) (Zheng and Wang 1999) was successfully modified and validated to simulate the fate and transport of fullerene nanoparticles $\mathrm{nC}_{60}$ in the subsurface field scale. Thus, this work aims at implementing the 1D
CFT model (equations 1-3) to multiple dimensions by further modifying our previous developed numerical simulator (Bai and Li 2012). MT3DMS is a multi-dimensional modelling tool for contaminant transport in groundwater, which is widely used to simulate advection, dispersion/diffusion and chemical reactions of contaminants in subsurface systems with various boundary conditions and external sources and sinks (Zheng and Wang 1999). This work utilised the nonequilibrium sorption model in MT3DMS as shown in the following equation:

$$
\rho_{\mathrm{b}} \frac{\partial S}{\partial t}=\beta\left(C-\frac{S}{K_{\mathrm{d}}}\right) .
$$

Here, $K_{\mathrm{d}}$ is the distribution coefficient for sorbed phase $\left[\mathrm{L}^{3} / \mathrm{M}\right]$ and $\beta$ is the first-order mass transfer rate between the dissolved and sorbed phases $[1 / \mathrm{T}]$. This non-equilibrium sorption module in MT3DMS could be modified to simulate the boron nanoparticles transport by setting $\beta$ as:

$$
\beta=n k_{\mathrm{att}} .
$$

Here, $K_{\mathrm{d}}$ in equation (4) was set to infinity so that the detachment rate did not have to be considered. Specifically, a subroutine for boron nanoparticles transport was developed and incorporated into the chemical reaction package of the MT3DMS program. The mass balance information for boron nanoparticles transport in the aqueous phase and attached phase was checked for each iteration.

The developed simulator for boron nanoparticles transport in subsurface was validated by comparing the results with the analytical solutions since there exist analytical solutions for 1D CFT model but not for $2 \mathrm{D}$ and $3 \mathrm{D}$, and the results from the simulator are consistent with 1D analytical solutions under a range of collision efficiency and flow velocities. Figure 1 lists one example of the validation. Here, the boron nanoparticles with a concentration of $0.05 \mathrm{mg} / \mathrm{l}$ and the particle size of $100 \mathrm{~nm}$ was injected into a 16-cm long column packed with sand. The sand packed in the column has an average diameter of $1.2 \mathrm{~mm}$ and porosity of 0.37 . The injection duration was $33.3 \mathrm{~min}$ with pore water velocity of $13.5 \mathrm{~m} / \mathrm{d}$ and the collision efficiency for boron nanoparticles was 0.01. As illustrated in figure 1 , the retention profile from the modifiedboron MT3DMS was consistent with the analytical solution very well. 


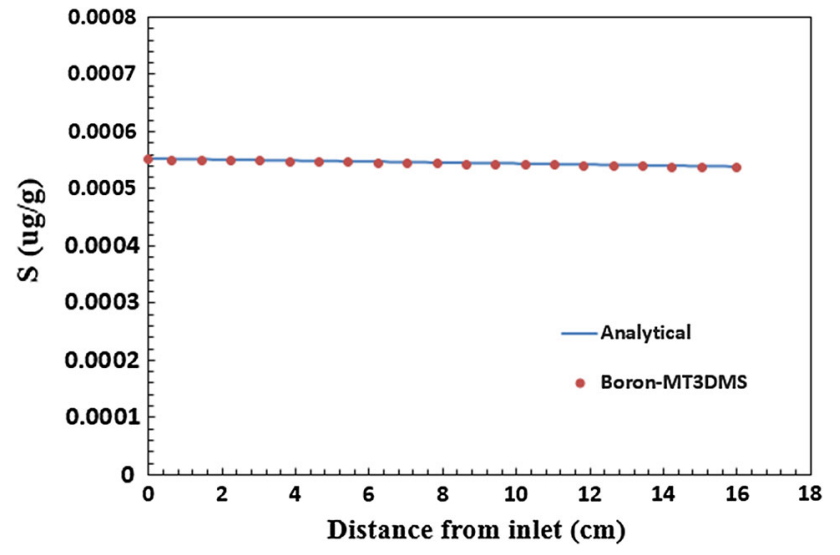

Figure 1. Comparison of boron-MT3DMS simulation result with the analytical solution for boron nanoparticles in a 1D sand column.

\section{Nanoparticle release scenarios}

Hypothetical scenarios for the release of boron nanoparticles and fullerene nanoparticles into the saturated porous media with an average porosity of 0.3 were investigated under different geological conditions, including assumed layered and heterogeneous cases. The fate of boron nanoparticles in a multi-dimensional subsurface would be simulated based on this newly developed simulator, while the fullerene nanoparticles transport was simulated using the simulator developed in our previous work (Bai and Li 2012). The study domain for nanoparticles transport would be targeted mainly in a rectangular region with length of $200 \mathrm{~cm}$ and depth of $54 \mathrm{~cm}$. A steady-state flow condition was attained by imposing a hydraulic gradient of 0.02 along the $x$-direction, with a constant head boundary condition (Type I Dirichlet BC) applied on both the left and right boundaries and no-flow boundary condition (Type II Neumann BC) applied on the bottom boundary. Both the boron nanoparticles and fullerene nanoparticles were released through the left boundary with a constant concentration of $10 \mathrm{ppm}$ for $2 \mathrm{~d}$. The simulation domain was divided into 36 layers with uniform grid size of $1.5 \mathrm{~cm}$ in the $z$-direction. To obtain a detailed information about nanoparticles distribution around the source zone, the domain was unevenly discretised along the $x$-direction into 38 columns, with finer grid size around the source zone, as detailed in table 1 . Six observation points, $\mathrm{O} 1, \mathrm{O} 2, \mathrm{O} 3, \mathrm{O} 4, \mathrm{O} 5$ and $\mathrm{O} 6$, were located at depths of $4.5,13.5,22.5,31.5,40.5$ and 49.5 $\mathrm{cm}$, respectively $(27 \mathrm{~cm}$ to the left boundary for them).
Discretisation of the simulation domain was same for both the layered case and heterogeneous case, while the hydraulic conductivity distribution was different between these two cases. In the layered scenario, there were six layers with uniform thicknesses of $9 \mathrm{~cm}$ for each layer, and the corresponding hydraulic conductivity distribution from top to bottom layers was 129.6, 12.96, 129.6, $1.296,12.96$ and $129.6 \mathrm{~m} / \mathrm{d}$, respectively. In the heterogeneous scenario, the background hydraulic conductivity $K$ equals $12.96 \mathrm{~m} / \mathrm{d}$, with four different slices arranged within the domain, as shown in figure 2. The hydraulic conductivity of the first slice was $K_{1}=K / 20$, which was located from 2 to $78.5 \mathrm{~cm}$ in the $x$-direction and 9 to $12 \mathrm{~cm}$ in the $z$-direction. The second slice's hydraulic conductivity was $K_{2}=20 K$, located from 12 to $78.5 \mathrm{~cm}$ in the $x$-direction and 19.5 to $22.5 \mathrm{~cm}$ in the $z$-direction. The third slice's hydraulic conductivity was $K_{3}=K / 10$, located from 2 to $54 \mathrm{~cm}$ in the $x$-direction and 31.5 to $34.5 \mathrm{~cm}$ in the $z$-direction. The fourth slice's hydraulic conductivity was $K_{4}=50 \mathrm{~K}$, located from 54 to $112 \mathrm{~cm}$ in the $x$-direction and 42 to $45 \mathrm{~cm}$ in the $z$-direction.

\section{Results and discussion}

\subsection{Layered scenario}

In a layered scenario, six observation points $\mathrm{O} 1$, $\mathrm{O} 2, \mathrm{O} 3, \mathrm{O} 4, \mathrm{O} 5$ and $\mathrm{O} 6$ were located at layers $1,2,3,4,5$ and 6 , respectively. The normalised aqueous-phase nanoparticles concentration $\left(C / C_{0}\right)$ was obtained at these observation points. Specifically, the results show that $C / C_{0}$ concentration of boron nanoparticles at $\mathrm{O} 2, \mathrm{O} 4$ and $\mathrm{O} 5$ were smaller than 0.01 , while at $\mathrm{O} 1, \mathrm{O} 3$ and $\mathrm{O} 6$ were $0.677,0.638$ and 0.651 , respectively. The difference in $C / C_{0}$ at different points is mainly due to various soil permeabilities of layers. There are three groups of hydraulic conductivity distribution in the study domain, with the first group of layers 1, 3 and 6 has the highest hydraulic conductivity of $129.6 \mathrm{~m} / \mathrm{d}$, the second group of layers 2 and 5 has hydraulic conductivity of $12.96 \mathrm{~m} / \mathrm{d}$ and the third group of layer 4 has the lowest hydraulic conductivity of $1.296 \mathrm{~m} / \mathrm{d}$. Higher hydraulic conductivity, higher soil permeability and higher velocity lead to higher normalised aqueous-phase nanoparticles concentration at the observation points for either 
Table 1. Parameters used in the model simulations.

\begin{tabular}{|c|c|c|}
\hline Dimensions & $200 \times 54$ & $\mathrm{~cm}$ \\
\hline $\mathrm{d} x$ & $\begin{array}{l}(1,0.5,0.5,0.5,0.5,1,1,1,2,2,3,3,4,4,6 \\
6,6,6,6,6,6,6,8,8,8,8,8,8,8,8,8,8,8 \\
8,8,8,8,8)\end{array}$ & $\mathrm{cm}$ \\
\hline $\mathrm{d} y$ & - & Dimensionless \\
\hline $\mathrm{d} z$ & $1.5 \times 36$ & $\mathrm{~cm}$ \\
\hline$\alpha_{\mathrm{L}}$ & 1 & $\mathrm{~cm}$ \\
\hline$\alpha_{\mathrm{T}} / \alpha_{\mathrm{L}}$ & 0.1 & Dimensionless \\
\hline Hydraulic gradient & 0.02 & Dimensionless \\
\hline Soil porosity & 0.3 & Dimensionless \\
\hline Release concentration $C_{0}$ & 10 & ppm \\
\hline Collision efficiency & 0.01 & Dimensionless \\
\hline Nanoparticle diameter & 100 & $\mathrm{~nm}$ \\
\hline
\end{tabular}
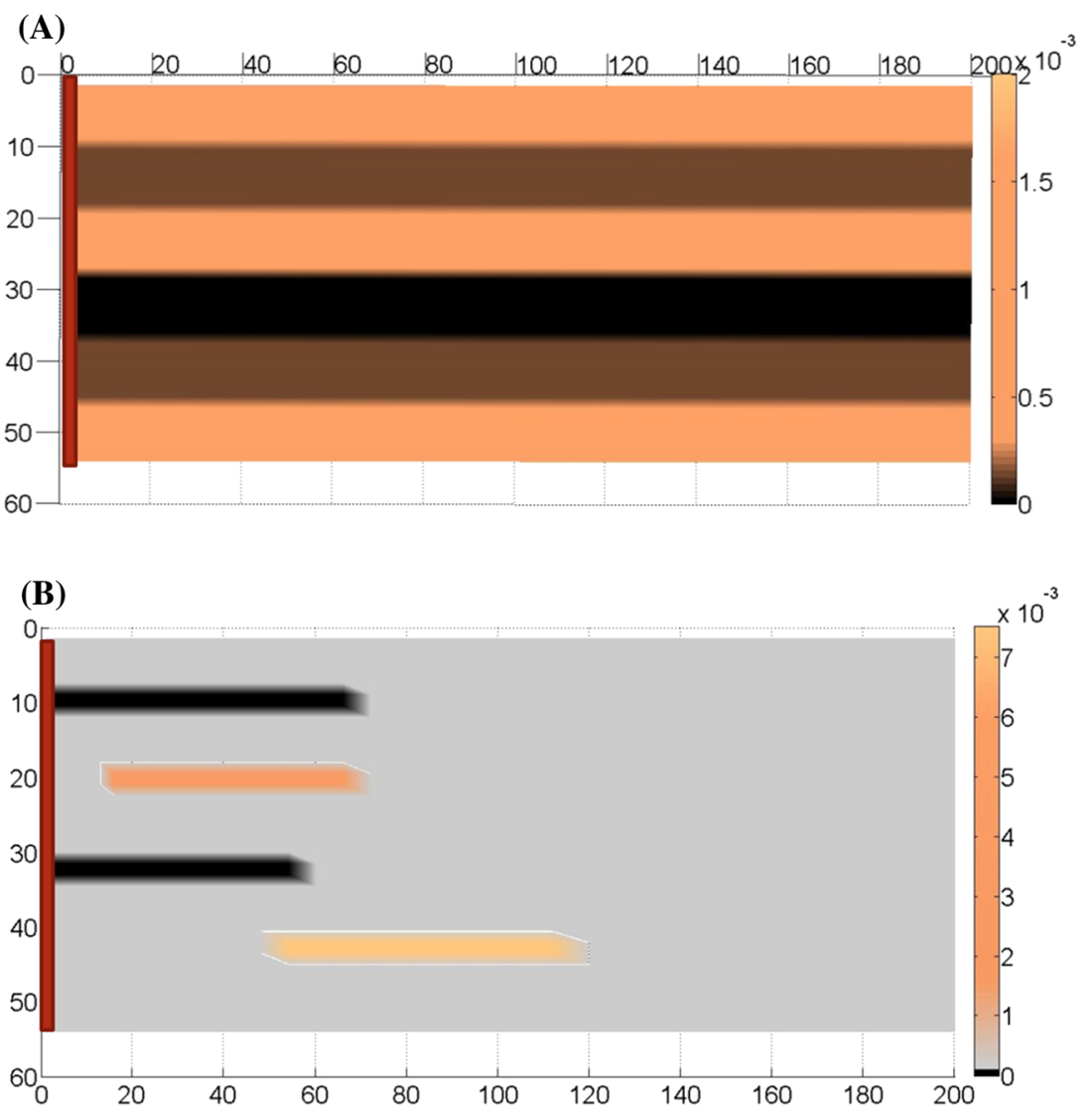

Figure 2. Hydraulic conductivity (unit: $\mathrm{m} / \mathrm{s}$ ) distribution for (a) layered case and (b) heterogeneous case. Here, red bar at the left boundary represents the source of nanoparticles.

boron nanoparticles or fullerene nanoparticles. Figure 3 lists the normalised aqueous-phase nanoparticles concentration at points $\mathrm{O} 1, \mathrm{O} 3$ and $\mathrm{O} 6$ for both nanoparticles. Even though the hydraulic conductivities of layers 1,3 and 6 were same, it is interesting to note that their $C / C_{0}$ were different, with $C / C_{0}$ at layer 1 larger than layer 6 and then layer 3 . In this study, this phenomenon was interpreted as different layers' interaction. Here, layer 1 was surrounded by layer 2 , which was then surrounded by layer 3 ; layer 3 was surrounded by layers 2 and 4, which has the lowest hydraulic conductivity; layer 6 was surrounded by layer 5 , which was surrounded by layer 4 . Overall, the surrounding layers for layer 3 have the lowest hydraulic conductivity, followed by layer 6 and then layer 1 , which lead to higher breakthrough curves for point O1, followed by O3 and then O6. This phenomenon 

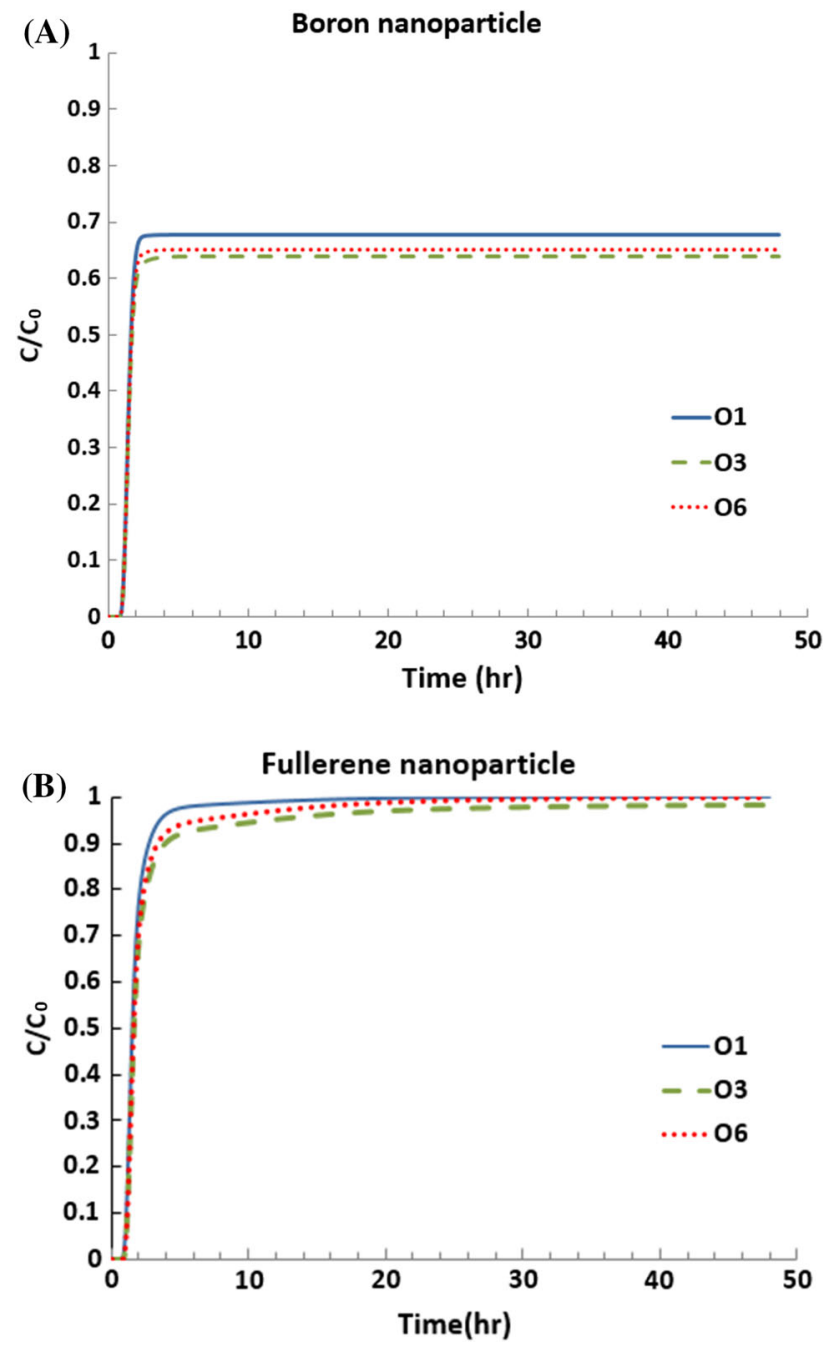

Figure 3. Breakthrough curves at observation points O1, O3 and O6 in layered case for (a) boron nanoparticles and (b) fullerene nanoparticles.

happens for both boron nanoparticles and fullerene nanoparticles, as shown in figure 3 ( $\mathrm{a}$ and $\mathrm{b}$ ).

By comparison, boron nanoparticles have lower normalised concentration than the fullerene nanoparticles. At the end of the simulation, the normalised concentration for fullerene nanoparticles reached $0.999,0.983$ and 0.999 at points $\mathrm{O} 1$, $\mathrm{O} 3$ and $\mathrm{O} 6$, respectively. The main reason is that boron nanoparticles follow the CFT model (Liu et al. 2009), which is different from the mechanistic model of fullerene nanoparticles. Compared to fullerene nanoparticles, boron nanoparticles are kept to deposit on soil medium since there was no maximum retention capacity, which resulted in more boron nanoparticles on soil and less boron nanoparticles in aqueous phase. The less number of boron nanoparticles in the aqueous phase would in turn lead to a smaller value of $C / C_{0}$ at corresponding points. Figure 4 shows the attached-phase concentration $(S)$ distribution in the study domain for these two nanoparticles. For boron nanoparticles, $S$ tends to accumulate at the inlet zone, as shown in figure 4(a). Specifically, there were two zones where boron nanoparticles were accumulated. One zone was the inlet of layer 2 with $S$ concentration of $49-53 \mu \mathrm{g} / \mathrm{g}$, and the other zone was the inlet of layer 5 with $S$ concentration of $48-53 \mu \mathrm{g} / \mathrm{g}$. The phenomenon that boron nanoparticles mainly accumulated at the inlet could be explained as that boron nanoparticles continuously attached to soil without a limit when the inlet portion's aqueous-phase boron concentration was highest. The combined effect of high aqueous-phase concentration $(C)$ and high attachment coefficient $\left(k_{\text {att }}\right)$ resulted in the high $S$ at the inlet of layers 2 and 5 . Here, even though the aqueous-phase boron concentration in layers 1, 3 and 6 was high, their attachment coefficient was low. Thus, most of the boron nanoparticles stay in the aqueous phase in layers 1, 3 and 6. Comparably, fullerene nanoparticles mainly accumulated at the interface of layers 3 and 4 . The main reason is that fullerene nanoparticles follow maximum retention capacity model, and layer 4's retention capacity is the highest one, while the aqueous-phase fullerene concentration in layer 3 was high. Thus, a lot of fullerene nanoparticles accumulate at the interface of layers 3 and 4 , with the highest $S$ reached being $24 \mu \mathrm{g} / \mathrm{g}$.

\subsection{Heterogeneous scenario}

To further investigate the impact of soil heterogeneity on the fate of boron nanoparticles in subsurface, this study simulated the transport and distribution of boron nanoparticles in a heterogeneous study domain. Figure 5 lists the aqueousphase and attached-phase boron nanoparticles distribution after $2 \mathrm{~d}$ of release of $10 \mathrm{mg} / \mathrm{l}$ boron nanoparticles. Similar to the layered case, boron nanoparticles mainly accumulated at the inlet, with the maximum attached-phase boron nanoparticles concentration reaching $63 \mu \mathrm{g} / \mathrm{g}$ at the inlet source zone near the second slice $K_{2}\left(K_{2}=20 \mathrm{~K}\right)$ after $2 \mathrm{~d}$ of release. Due to the existence of low permeable zones $K_{1}$ and $K_{3}\left(K_{1}=K / 20\right.$ and $K_{3}=$ $K / 10)$, boron nanoparticles bypassed these two low permeable zones $\left(K_{1}\right.$ and $\left.K_{3}\right)$. Thus, at these two zones of $K_{1}$ and $K_{3}$, either the aqueous-phase or attached-phased boron nanoparticles concentration was very little, as shown in figure 5 . However, some boron nanoparticles could reach $K_{2}$ zone after $2 \mathrm{~d}$ 

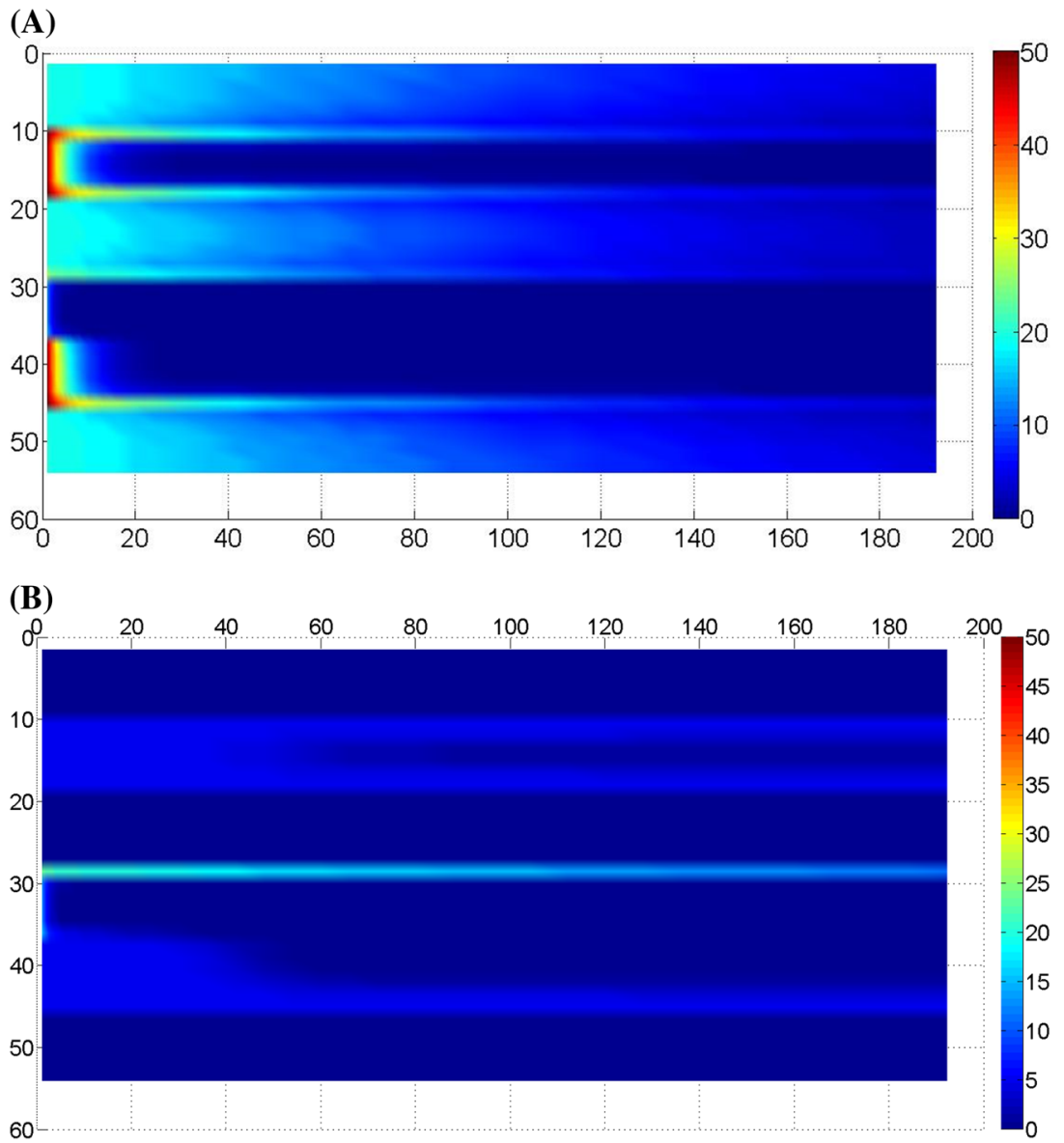

Figure 4. Attached concentration distribution (unit: $\mu \mathrm{g} / \mathrm{g}$ ) in the $2 \mathrm{D}$ layered domain for (a) boron nanoparticles and (b) fullerene nanoparticles.

of release, which was higher permeable zone than $K_{1}$ and $K_{3}$, and closer to the source zone compared to $K_{4}$ zone.

\subsection{Effects of release concentration $C_{0}$}

In this section, the effect of boron nanoparticles release concentration is investigated by varying the initial release concentration across two orders of magnitude. In this study, the release concentration of 1 and $100 \mathrm{ppm}$ for boron nanoparticles is discussed and compared for each of the two scenarios. In general, the higher release concentration leads to a higher contamination level of boron nanoparticles, which happens for either layered or heterogeneous scenario. Figure 6 illustrates that the aqueous-phase boron nanoparticles concentration for $C_{0}$ equals 10 and $100 \mathrm{ppm}$, respectively. Obviously, the higher release concentration led to the higher concentration distribution in the whole domain. For the layered scenario, the release concentration of $100 \mathrm{ppm}$ led to the observed concentration of $67.694,63.824$ and $65.138 \mathrm{ppm}$ at points of $\mathrm{O} 1, \mathrm{O} 3$ and $\mathrm{O} 6$, respectively. In comparison, an initial release concentration of 1 ppm only led to the concentration of 0.67656 , 0.63785 and $0.65101 \mathrm{ppm}$ at points of $\mathrm{O} 1, \mathrm{O} 3$ and O6, respectively. Besides, the higher release concentration caused standardised concentration $\left(C / C_{0}\right)$ to increase slightly faster at the corresponding observation points, which could be explained as that soil medium was not so instant to attach all the released boron nanoparticles in the subsurface at a time. Also, the phenomenon of different layers' interaction happens for release concentration of 1 and $100 \mathrm{ppm}$. In other words, the standardised concentration at $\mathrm{O} 1$ was larger than that of $\mathrm{O} 6$, and then $\mathrm{O} 3$, even though layers 1,3 and 6 have the same hydraulic conductivity. The higher release concentration also lead to higher concentration of boron nanoparticles attached to the soil medium. When $C_{0}$ equals $100 \mathrm{ppm}$, the maximum attached-phase boron nanoparticles concentration was $530 \mu \mathrm{g} / \mathrm{g}$, while the maximum boron nanoparticles attached to soil was only $5.3 \mu \mathrm{g} / \mathrm{g}$ when $C_{0}$ was $1 \mathrm{ppm}$. Similarly, 

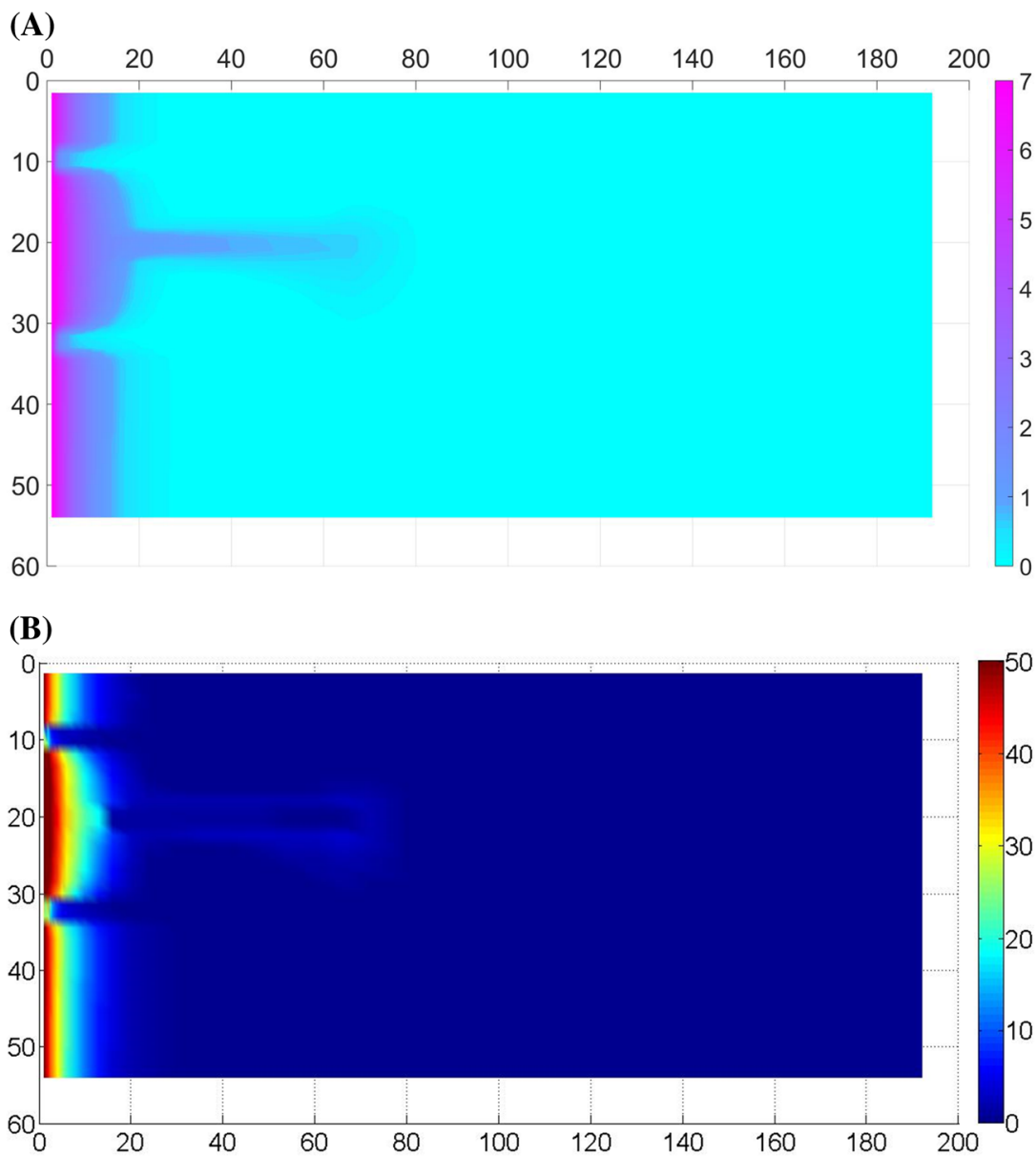

Figure 5. Boron nanoparticles concentration distribution in the heterogeneous domain: (a) aqueous-phase concentration (unit: $\mathrm{mg} / \mathrm{l}$ ) and (b) attached-phase concentration (unit: $\mu \mathrm{g} / \mathrm{g}$ ).

there were two zones where boron nanoparticles accumulated. One zone was the inlet of layer 2 and the other zone was the inlet of layer 5. For the heterogeneous scenario, the higher release concentration also lead to higher level of boron nanoparticles contamination in the saturated porous media. Also, boron nanoparticles bypassed two low permeable zones of $K_{1}$ and $K_{3}$, but reached high permeable zone of $K_{2}$ after 2 d of release. Most of the boron nanoparticles are attached to the soil medium around the source zone.

\subsection{Effects of porosity}

The porosity of soil depends on several factors, including packing density, cementing, etc. To explore the impact of soil porosity on the fate and transport of boron nanoparticles in saturated porous media, the sensitivity analysis was conducted for soil porosity of $0.2,0.3$ and 0.4 for both layered and heterogeneous scenarios. The result showed that the higher soil porosity increased boron nanoparticles attachment rate and thus results in more retention and lower mobility of boron nanoparticles in saturated porous media. Figure 7 provides a comparison of the distribution of attached-phase boron nanoparticles across the study domain for porosity of 0.2 and 0.4 for a layered scenario. Specifically, the maximum attachedphase boron nanoparticles concentration reached 27 and $81 \mu \mathrm{g} / \mathrm{g}$ for porosity of 0.2 and 0.4 , respectively. After $2 \mathrm{~d}$ of release, the observed aqueousphase concentration of boron nanoparticles was $8.302,7.9714$ and $8.0852 \mathrm{mg} / \mathrm{l}$ at observation points of $\mathrm{O} 1, \mathrm{O} 3$ and $\mathrm{O} 6$, respectively, when the soil porosity was 0.2 . On the other hand, when the soil porosity was 0.4 , the observed aqueous-phase concentration was $5.0846,4.7326$ and $4.8519 \mathrm{mg} / \mathrm{l}$ at O1, O3 and O6, respectively. Obviously, the higher the porosity, the tendency for boron nanoparticles to attach to soil is higher, which further results in more boron nanoparticles attached to the porous media and less boron nanoparticles remain in the aqueous phase. The influence of soil porosity 

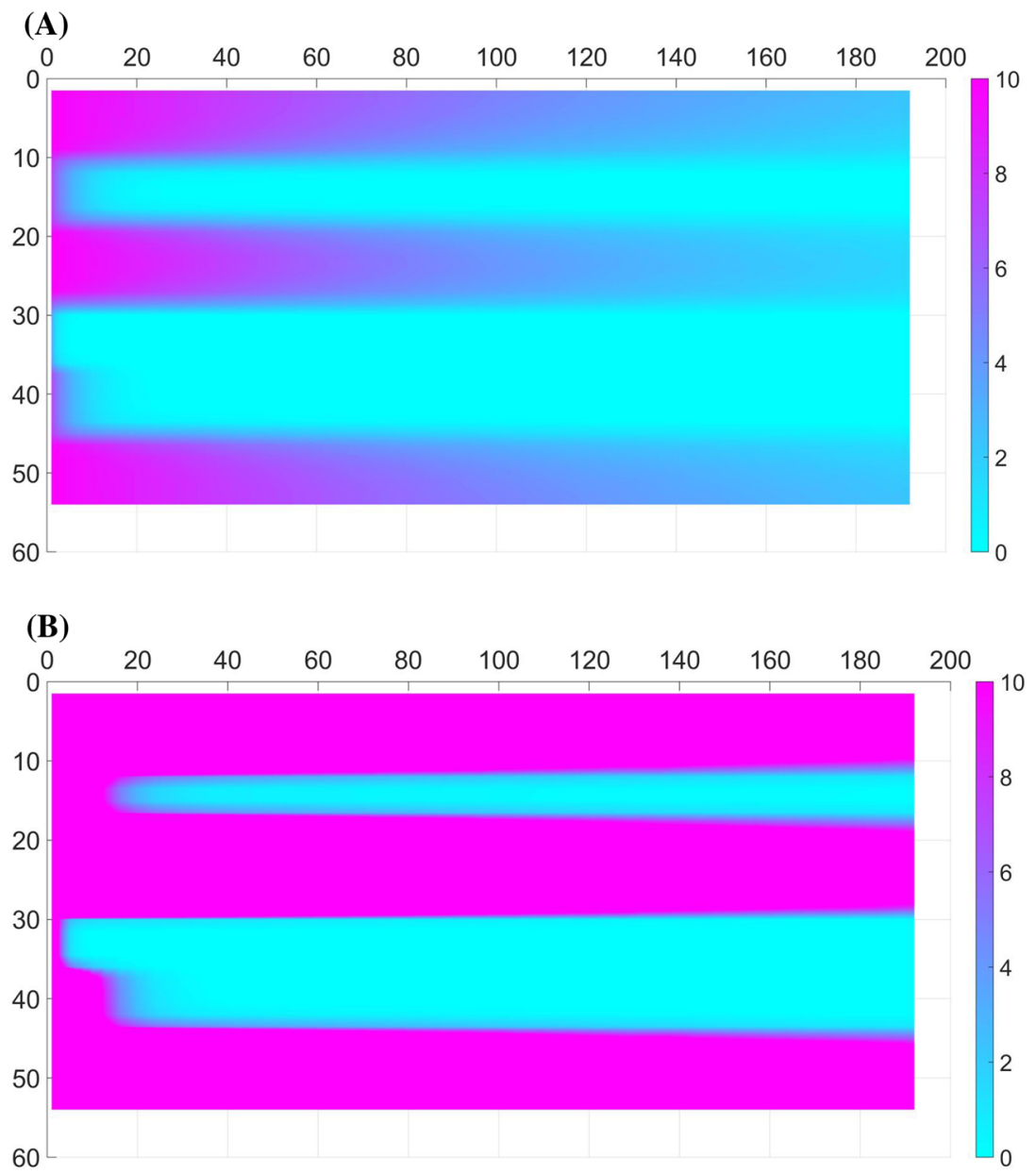

Figure 6. Aqueous-phase boron nanoparticles concentration (unit: $\mathrm{mg} / \mathrm{l})$ distribution in the layered domain: $(\mathbf{a}) C_{0}=10 \mathrm{ppm}$ and (b) $C_{0}=100 \mathrm{ppm}$.

was also examined for the heterogeneous scenario. Similarly, higher porosity results in higher attachment rate and more attached concentration and less aqueous-phase boron nanoparticles concentration. The maximum attached-phase concentration for boron nanoparticles in the study domain was 33,63 and $97 \mu \mathrm{g} / \mathrm{g}$ for porosities of $0.2,0.3$ and 0.4 , respectively.

\subsection{Effects of aggregate size $d p$}

To further investigate the impact of boron nanoparticles aggregate size, sensitivity analyses were conducted for particle size of 10, 100 and $1000 \mathrm{~nm}$ for both the layered and heterogeneous scenarios. In both scenarios, the larger the boron nanoparticles size was, the higher the mobility was. Figure 8 provided a comparison of boron nanoparticles concentration in the aqueous phase for diameters of 10 and $1000 \mathrm{~nm}$ in the heterogeneous scenario. Obviously, the larger nanoparticle leads to higher mobility, and thus less attachment on soil. After $2 \mathrm{~d}$ of release, boron nanoparticles with a diameter of $1000 \mathrm{~nm}$ reached higher permeable zones of $K_{2}$ and even $K_{4}$, while boron nanoparticles still bypassed low permeable zones of $K_{1}$ and $K_{3}$. In addition, the maximum boron nanoparticles concentration in the aqueous phase was $5.9,7.9$ and $9.4 \mathrm{mg} / \mathrm{l}$ for diameters of 10 , 100 and $1000 \mathrm{~nm}$, respectively. The influence of $\mathrm{dp}$ on the mobility of boron nanoparticles was also examined for the layered scenario. The smaller the particle diameter, the higher will be the attachment and thus lower mobility. The corresponding maximum attached-phase concentration was 120 and $27 \mu \mathrm{g} / \mathrm{g}$ for diameters of 10 and $1000 \mathrm{~nm}$. After 2 d's release, the observed aqueousphase concentrations for particle diameter of $10 \mathrm{~nm}$ were $1.0748,1.0110$ and $1.0286 \mathrm{mg} / \mathrm{l}$, at points of O1, O3 and O6, respectively. For particle diameter of $1000 \mathrm{~nm}$, the concentrations were 8.9652 , 8.6264 and $8.8028 \mathrm{mg} / \mathrm{l}$ at corresponding points 

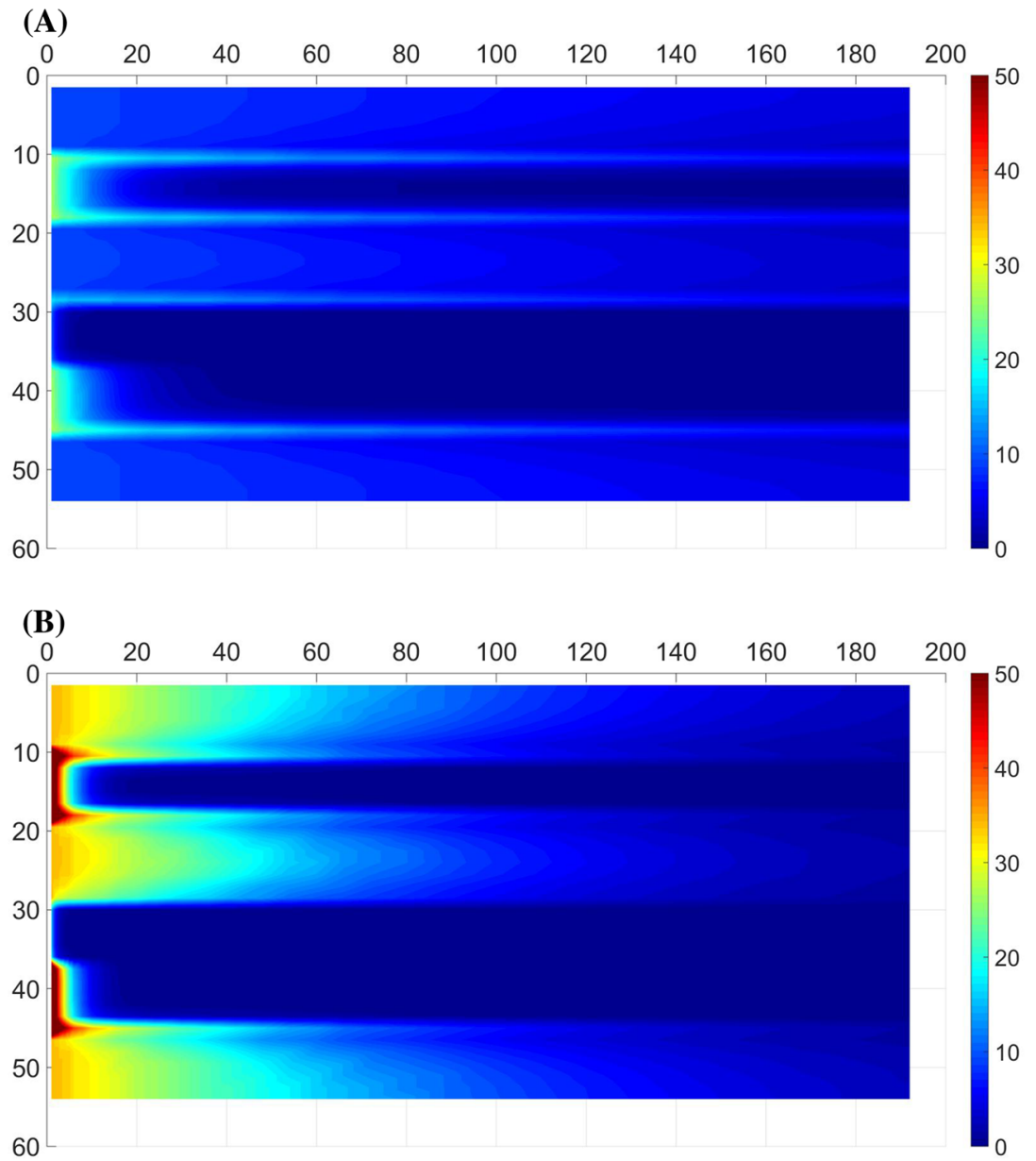

Figure 7. Attached-phase boron nanoparticles concentration (unit: $\mu \mathrm{g} / \mathrm{g}$ ) distribution in the layered domain: (a) porosity $=0.2$ and $(\mathbf{b})$ porosity $=0.4$.

of $\mathrm{O} 1, \mathrm{O} 3$ and $\mathrm{O} 6$. The main reason is that the smaller nanoparticle size led to higher $\eta_{0}$, and thus higher attachment rate (equation 3 ) and lower mobility.

\section{Summary and conclusions}

In this work, a multi-dimensional numerical simulator for the fate and transport of boron nanoparticles in saturated porous media was first developed and validated. Hypothetical scenarios for the release of boron nanoparticles into the $2 \mathrm{D}$ domain, including a layered domain and a heterogeneous domain, were explored. The results showed that nanoparticles tend to have high mobility in high permeable soil layers, which lead to higher normalised aqueous-phase concentration $C / C_{0}$ at corresponding observation points. In general, boron nanoparticles have lower normalised aqueous-phase concentration than fullerene nanoparticles, since boron nanoparticles continuously deposit on soil, which in turn leads to less concentration in the aqueous phase and then smaller $C / C_{0}$. For boron nanoparticles, even though the hydraulic conductivities for layers 1, 3 and 6 were same, their normalised aqueous-phase concentrations were different. Standardised concentration $C / C_{0}$ at layer 1 was larger than that of layer 6 , and then layer 3 . This phenomenon was interpreted as 'interface interaction' since the surrounding hydraulic conductivity of layer 3 was the smallest one. Different layers' interface interaction was the reflection of the fact that layers with small $K$ have lower $C / C_{0}$. This phenomenon of different layers' interface interaction happens for both boron nanoparticles and fullerene nanoparticles in the saturated porous media. Besides, boron nanoparticles tend to accumulate at the inlet zones, where the aqueousphase boron nanoparticles concentration was high and they keep deposited onto soil. Comparably, fullerene nanoparticles mainly accumulate at the interface of layers 3 and 4, where the aqueous-phase concentration was high and the retention capacity 

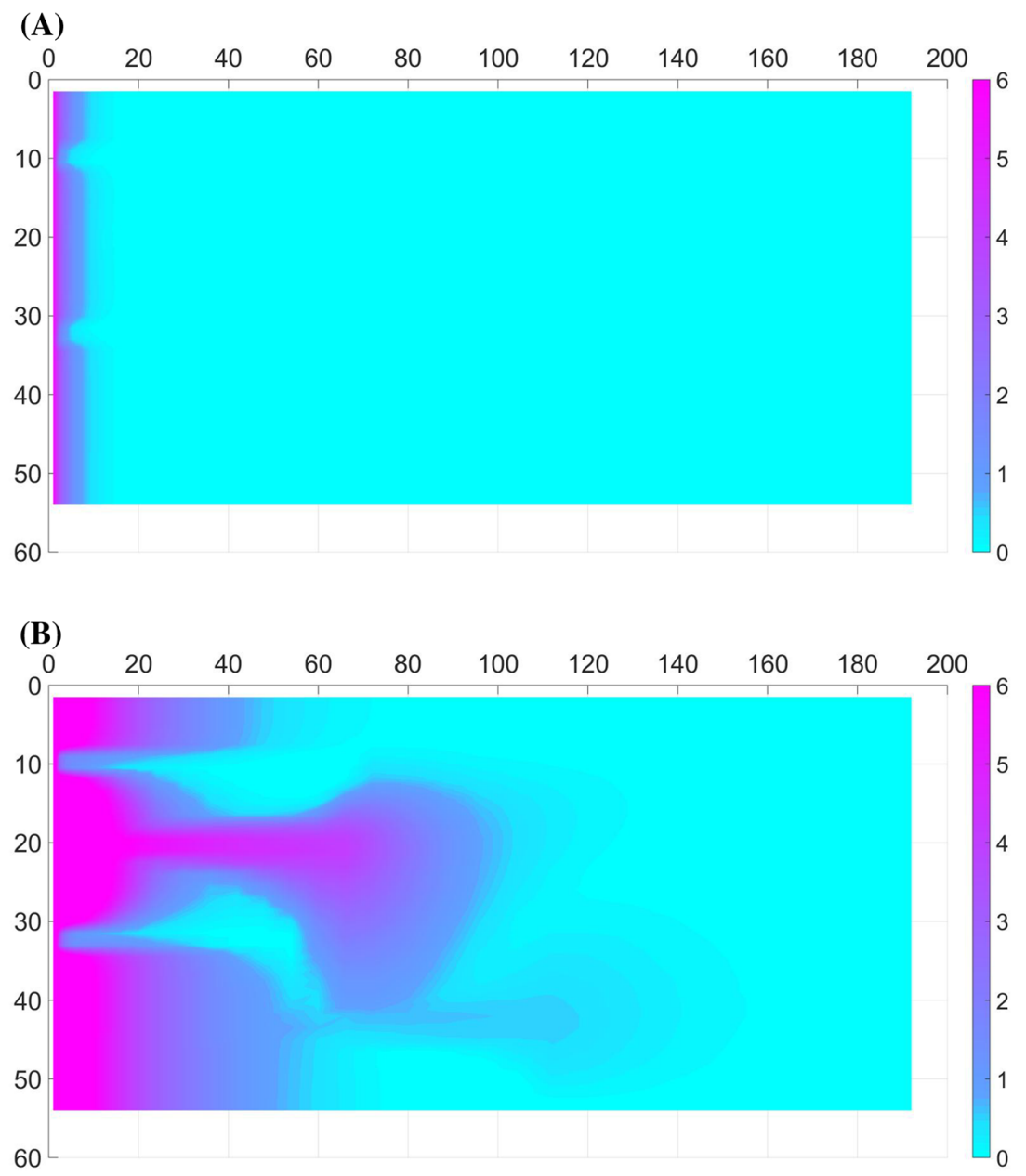

Figure 8. Aqueous-phase boron nanoparticles concentration (unit: $\mathrm{mg} / \mathrm{l}$ ) distribution in the heterogeneous domain: (a) $\mathrm{dp}=10 \mathrm{~nm}$ and $(\mathbf{b}) \mathrm{dp}=1000 \mathrm{~nm}$.

was also high. In the heterogeneous scenario, boron nanoparticles also accumulated at the inlet zones. Furthermore, boron nanoparticles bypassed the two low permeable zones ( $K_{1}$ and $\left.K_{3}\right)$, but reached high permeable zone $K_{2}$, which was comparably near the source than $K_{4}$ zone.

For the conditions investigated in this study, boron nanoparticles transport was sensitive to release concentration, soil porosity and boron nanoparticles aggregate size. The higher release concentration led to higher contamination of boron nanoparticles in the study domain. The higher soil porosity increased the attachment rate of boron nanoparticles on soil porous media, thus results in more retention and lower mobility. The smaller the nanoparticle size, the higher will be the attachment and thus lower mobility. The effects of release concentration, soil porosity and nanoparticles aggregate size on the transport of boron nanoparticles in saturated porous media were consistent for both scenarios. The mass balance discrepancies were
$<0.1 \%$ for all the simulations discussed in this study.

\section{Acknowledgements}

The authors thank Dr Yusong Li at University of Nebraska-Lincoln for his helpful suggestions and discussions. This research was supported by the Project of the National Natural Science Foundation of China (51705078), Fuzhou University Research Funding Project (XRC-1546) and Fujian Provincial Department of Education Fund Project (JAT160050).

\section{References}

Bai C, Eskridge K M and Li Y 2013 Analysis of the fate and transport of $\mathrm{nC}_{60}$ nanoparticles in the subsurface using response surface methodology; J. Contam. Hydrol. 152 60-69. 
Bai C and Li Y 2012 Modeling the transport and retention of $\mathrm{nC}_{60}$ nanoparticles in the subsurface under different release scenarios; J. Contam. Hydrol. 136 43-55.

Bai C and Li Y 2014 Time series analysis of contaminant transport in the subsurface: Applications to conservative tracer and engineered nanomaterials; J. Contam. Hydrol. 164 153-162.

Barlebo H C, Hill M C and Rosbjerg D 2004 Investigating the Macrodispersion Experiment (MADE) site in Columbus, Mississippi, using a three-dimensional inverse flow and transport model; Water Resour. Res. 40(4) W04211.

Chen K L and Elimelech M 2006 Aggregation and deposition kinetics of fullerene (C-60) nanoparticles; Langmuir 22(26) 10994-11001.

Chen K L and Elimelech M 2007 Influence of humic acid on the aggregation kinetics of fullerene (C-60) nanoparticles in monovalent and divalent electrolyte solutions; J. Colloid Interface Sci. $\mathbf{3 0 9 ( 1 )} 126-134$.

Cheng X K, Kan A T and Tomson M B 2005 Study of C-60 transport in porous media and the effect of sorbed C-60 on naphthalene transport; J. Mater. Res. 20(12) 3244-3254.

Christ J A, Lemke L D and Abriola L M 2005 Comparison of two-dimensional and three-dimensional simulations of dense nonaqueous phase liquids (DNAPLs): Migration and entrapment in a nonuniform permeability field; Water Resour. Res. 41(1) W01007.

Cullen E, O'Carroll D M, Yanful E K and Sleep B 2010 Simulation of the subsurface mobility of carbon nanoparticles at the field scale; Adv. Water Resour. 33(4) 361-371.

Espinasse B, Hotze E M and Wiesner M R 2007 Transport and retention of colloidal aggregates of C-60 in porous media: Effects of organic macromolecules, ionic composition, and preparation method; Environ. Sci. Technol. 41(21) 7396-7402.

Hotze E M, Phenrat T and Lowry G V 2010 Nanoparticle aggregation: Challenges to understanding transport and reactivity in the environment; J. Environ. Qual. 39(6) 1909-1924.

Joo S H and Zhao D 2016 Environmental dynamics of metal oxide nanoparticles in heterogeneous systems: A review; J. Hazard. Mater. 322 29-47.

Kamat J P, Devasagayam T P A, Priyadarsini K I and Mohan H 2000 Reactive oxygen species mediated membrane damage induced by fullerene derivatives and its possible biological implications; Toxicology 155(1-3) 55-61.

Li Y S, Wang Y G, Pennell K D and Abriola L M 2008 Investigation of the transport and deposition of fullerene (C60) nanoparticles in quartz sands under varying flow conditions; Environ. Sci. Technol. 42(19) 7174-7180.

Liu X, Wazne M, Christodoulatos C and Jasinkiewicz K L 2009 Aggregation and deposition behavior of boron nanoparticles in porous media; J. Colloid Interface Sci. 330(1) 90-96.

LuxResearch 2009 Nanomaterials state of the market Q1 2009: Cleantech's dollar investments, Penny Returns; from https://members.luxresearchinc.com/research/ report $/ 2386$.
Lyon D Y, Adams L K, Falkner J C and Alvarez P J J 2006 Antibacterial activity of fullerene water suspensions: Effects of preparation method and particle size; Environ. Sci. Technol. 40(14) 4360-4366.

Ma H, Pedel J, Fife P and Johnson W P 2009 Hemispheres-in-cell geometry to predict colloid deposition in porous media; Environ. Sci. Technol. 43(22) 85738579.

Mauter M S and Elimelech M 2008 Environmental applications of carbon-based nanomaterials; Environ. Sci. Technol. 42(16) 5843-5859.

Nakajima N, Nishi C, Li F M and Ikada Y 1996 Photoinduced cytotoxicity of water-soluble fullerene; Fullerene Sci. Technol. 4(1) 1-19.

Oberdorster E 2004 Manufactured nanomaterials (fullerenes, C-60) induce oxidative stress in the brain of juvenile largemouth bass; Environ. Health Perspect. 112(10) 10581062.

Saiers J E and Ryan J N 2005 Colloid deposition on nonideal porous media: The influences of collector shape and roughness on the single-collector efficiency; Geophys. Res. Lett. 32(21) 1-5.

Sayes C M, Gobin A M, Ausman K D, Mendez J, West $\mathrm{J}$ L and Colvin V L 2005 Nano-C-60 cytotoxicity is due to lipid peroxidation; Biomaterials 26(36) 7587 7595 .

Tufenkji N and Elimelech M 2004 Correlation equation for predicting single-collector efficiency in physicochemical filtration in saturated porous media; Environ. Sci. Technol. 38(2) 529-536.

UNEP 2007 Chapter 7: Emerging challenges-nanotechnology and the environment; In: GEO year book, United Nations Environment Programme Division of Early Warning and Assessment, Nairobi, pp. 61-70.

Wang Y G, Li Y S, Abriola L M and Pennell K D 2008a Transport and retention of fullerene (C60) nanoparticles in natural soils; Eos Trans. AGU, Fall Meet. Suppl. 89(53) H43E-1059.

Wang Y G, Li Y S and Pennell K D 2008b Influence of electrolyte species and concentration on the aggregation and transport of fullerene nanoparticles in quartz sands; Environ. Toxicol. Chem. 27(9) 18601867.

WWICS 2012 Nanotechnology consumer product inventory; from http://www.nanotechproject.org/inventories/ consumer.

Yang K, Zhu L Z and Xing B S 2006 Adsorption of polycyclic aromatic hydrocarbons by carbon nanomaterials; Environ. Sci. Technol. 40(6) 1855-1861.

Yao K M, Habibian M M and Omelia C R 1971 Water and waste water filtration - Concepts and applications; Environ. Sci. Technol. 5 (11) 1105-1112.

Zheng C and Wang P P 1999 MT3DMS: A modular threedimensional multi-dimensional multi-species model for simulation of advection, dispersion and chemical reactions of contaminants in groundwater systems: Documentation and user's guide, U.S.A.E.R.a.D. Center, Vicksburg, MS. 